\title{
Appendiceal mucocele due to mucinous adenoma diagnosed by computed tomography colonography
}

\section{Nicola Flor, Gianpaolo Carrafiello}

Azienda Servizi Socio Territoriali Santi Paolo e Carlo; Università degli Studi di Milano, Milan, Italy

An asymptomatic 50-year-old man underwent a low-dose computed tomography colonography (CTC) for screening purpose. The CTC examination was negative for colonic mass or polyps, but axial and coronal two-dimensional CTC images (Fig. 1) showed a large elongated low-attenuation mass in the expected region of the appendix, in line with an appendiceal mucocele. Three-dimensional CTC endoluminal images (Fig. 2) showed the mass bulging into the cecal lumen.

The patient underwent appendectomy with partial right hemicolectomy and is in good health. Pathology confirmed an appendiceal mucocele from mucinous adenoma, and in particular the histological examination revealed a low-grade mucinous appendiceal neoplasm with negative regional lymph nodes and with no signs of mucinous peritoneal carcinomatosis.

This case shows the crucial role of CTC in the diagnosis of an unknown submucosal disease [1], potentially malignant if not early diagnosed [2,3]; that diagnosis would not have been made if the patient had had an optical colonoscopy or a double contrast barium enema instead of CTC, since both tests fail to evaluate extraluminal areas.

Unità Operativa di Radiologia Diagnostica e Interventistica, Azienda Servizi Socio Territoriali Santi Paolo e Carlo; Dipartimento di Scienze della Salute, Università degli Studi di Milano, Milan, Italy

Conflict of Interest: None

Correspondence to: Nicola Flor, Dipartimento di Scienze della Salute, Università degli Studi di Milano, Via di Rudinì 8, 20142 Milan, Italy, Tel.: +39 0281844307, Fax: +39 0281844652,

e-mail: nicola.flor@unimi.it

Received 23 September 2016; accepted 11 October 2016; published online 4 November 2016

DOI: https://doi.org/10.20524/aog.2016.0103

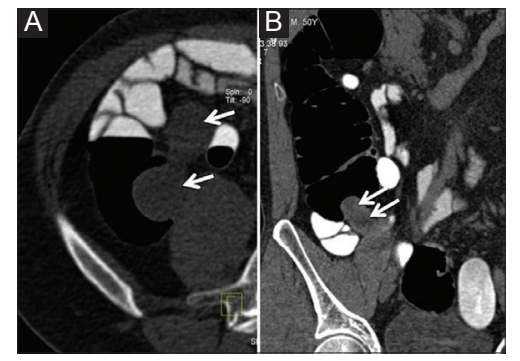

Figure 1 Axial prone (A) and coronal reformation (B) computed tomography colonography images show a dilated appendix appearing as a smooth-walled, homogeneous low-density tubular structure (arrows in A and B) protruding into the cecum lumen
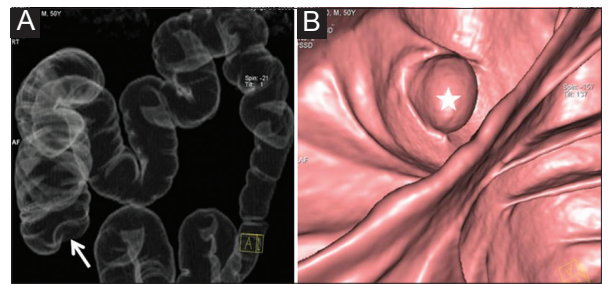

Figure $23 \mathrm{D}$ color map image (A) shows a cecum smooth filling defect (arrow). 3D endoluminal view (B) demonstrates a smooth mass (asterisk) protruding into the cecum

\section{References}

1. Pickhardt PJ, Levy AD, Rohrmann CA Jr, Kende AI. Primary neoplasms of the appendix: radiologic spectrum of disease with pathologic correlation. Radiographics 2003;23:645-662.

2. Jacobs JE, Balthazar EJ. Diseases of the appendix. In: Textbook of gastrointestinal radiology, 3rd edn. Saunders: Philadelphia; 2008, pp. 1039-1069.

3. Hines JJ, Paek GK, Lee P, Wu L, Katz DS. Beyond appendicitis; radiologic review of unusual and rare pathology of the appendix. Abdom Radiol (NY) 2016;41:568-581. 